\title{
UN MODELO CAUSAL SOBRE LOS ANTECEDENTES Y CONSECUENTES DEL ESTRES DE ROL EN PROFESIONALES DE LA SALUD
}

\author{
María Jesús Bravo, Rosario Zurriaga, José María Peiró y Pilar González. \\ Area de Psicología Social. \\ Universitat de Valencia.
}

\section{RESUMEN}

En el presente trabajo se somete a contrastación empírica un modelo causal sobre los antecedentes y las consecuencias del estrés de rol en profesionales sanitarios. La muestra utilizada es de 178 profesionales sanitarios (médicos y personal de enfermería) que desempeñan su rol en hospitales, clínicas y Centros de Atención Primaria. Para examinar el modelo se ha utilizado el paquete estadístico LISRELL VII. Los resultados obtenidos muestran que la estandarización de objetivos tiene un efecto directo y negativo sobre el conflicto de rol y un efecto directo y positivo sobre la claridad de rol y la satisfacción laboral; el conflicto de rol no tiene ningún efecto directo sobre la satisfacción laboral; mientras que la claridad de rol tiene un efecto directo y positivo sobre la satisfacción laboral y negativos sobre la tensión. Por último, la tensión laboral tiene un efecto directo y positivo sobre la propensión al abandono, no siendo significativo el efecto de la satisfacción laboral sobre esta última variable.

Palabras clave: estrés de rol, profesionales de la salud, modelo causal. 


\section{ABSTRACT}

In this paper we propose a causal model of antecedents and consequences of rol stress in health proffesionals. The sample was composed by 178 health professionals (physicians and nurses) who were working in hospitals, clinics and primary health care centres. The model was tested in this study using LISREL VII. The findings suggest that the standarization has a significant, direct and negative effect on role conflict and a significant, direct and positive effect on role clarity and job satisfaction. In the other hand, role conflict has no direct effect on job satisfaction, but role clarity has a significant, direct and positive effect on tension. Finally, job tension has a significant, direct and positive effect on propensity to leave, although satisfaction has no effect on this last aspect.

Key words: role stress, health professionals, causal model.

\section{INTRODUCCION}

El estrés de rol en las organizaciones ha sido un tema que ha generado una extensa investigación desde que Kahn et al. (1964) desarrollaron su modelo teórico. En este sentido, las fuentes de estrés más citadas en la literatura son: el conflicto y la ambigüedad de rol, la sobrecarga de rol, la infrautilización de habilidades, los recursos inadecuados y la escasa participación. Estos estresores predicen una serie de efectos actitudinales y conductuales negativos, tales como la insatisfacción laboral, la tensión, la propensión al abandono o un bajo desempeño (Cummings, 1990).

La mayoría de los estudios se han centrado en las correlaciones entre los estresores de rol y sus correlatos hipotetizados, utilizándose posteriormente diversos meta-análisis para evaluar la fuerza y dirección de dichos correlatos (Fisher y Gitelson, 1983; Jackson y Schuler, 1985). En ellos, como señala Lloret (1991), se aporta, aunque con ciertas reservas, evidencia empírica a favor del modelo teórico propuesto por Kahn et al. (1964).

Solamente a partir de la segunda mitad de los años 70 se han desarrollado estudios que, utilizando técnicas de modelado causal multivariado, han permitido poner a prueba hipótesis de causalidad entre el estrés de rol y sus antecedentes y consecuentes (Oliver y Brief, 1977-1978; Bedeian y Armenakis, 1981; Jackson, 1983, 1984; Peiró et al., 1985; Kemery et al., 1985; Fry et al., 1986; Gonzalez-Romá et al., 1989; Lloret, 1991). 
Algunos de estos trabajos se han realizado con muestras de profesionales de la salud. De ellos, destacaremos los de Bedeian y Armenakis (1981); Kemery et al. (1985); González Romá et al. (1989) y Lloret (1991).

El estudio de Bedeian y Armenakis (1981), realizado con una muestra compuesta por 202 enfermeras, puede considerarse clásico en esta línea. Estos autores hipotetizan que el conflicto y la ambigüedad de rol tienen efectos directos de carácter positivo sobre la tensión laboral y la propensión al abandono y negativos sobre la satisfacción laboral. Asimismo hipotetizan efectos indirectos del conflicto y la ambigüedad de rol sobre la satisfacción laboral a través de la tensión y sobre la propensión al abandono a través de la tensión y la satisfacción laboral. Por otra parte se hipotetizan también efectos directos negativos de la tensión sobre la satisfacción laboral e indirectos sobre la propensión al abandono, mientras que la satisfacción laboral tiene un efecto directo negativo sobre la propensión al abandono.

Los resultados obtenidos, utilizando la técnica de path analysis, han corroborado algunas de sus hipótesis de partida, y se pueden resumir en los siguientes puntos (Lloret, 1991):

- La ambigüedad de rol tiene efectos directos positivos sobre la tensión laboral y negativos sobre la satisfacción laboral, mientras que sus efectos directos sobre la propensión al abandono son insignificantes.

- El conflicto de rol tiene efectos directos positivos sobre la tensión y negativos sobre la satisfacción laboral. Al igual que ocurría con la ambigüedad de rol, los efectos sobre la propensión al abandono son también insignificantes.

- La tensión tiene efectos directos y negativos sobre la satisfacción.

- La satisfacción tiene efectos directos y negativos sobre la propensión al abandono.

Estos autores concluyen que la tensión es el predictor de mayor impacto de la satisfacción laboral, lo que sugiere que el conflicto y la ambigüedad de rol tienen efectos indirectos sobre la satisfacción laboral a través de la tensión. Por otra parte, la satisfacción laboral resulta ser una variable de utilidad para predecir la propensión al abandono.

Los trabajos de Kemery et al. (1985) y de González-Romá et al. (1989), realizados también con muestras de profesionales de la salud, son replicaciones 
del modelo propuesto por Bedeian y Armenakis (1981). En ambos se han encontrado patrones de influencia diferentes para el conflicto y la ambigüedad de rol, ya que la ambigüedad influye únicamente sobre la tensión, mientras que el conflicto de rol influye también sobre la satisfacción laboral e indirectamente sobre la propensión al abandono a través de la satisfacción. Estos resultados no concuerdan plenamente con los obtenidos por Bedeian y Armenakis (1981).

Se observan también concordancias en los resultados obtenidos respecto a la tensión laboral en los estudios de Bedeian y Armenakis (1981) y Kemery et al. (1985), ya que ésta influye de forma directa sobre la satisfacción laboral, mientras que en el trabajo de González-Romá et al. (1989), dicha influencia no se observa.

Posteriormente, Lloret (1991) realizó un estudio cuyo objetivo era clarificar el proceso de estrés de rol, analizando sus antecedentes y consecuencias a través de técnicas de análisis correlacional multivariado (utilizando modelado causal), en profesionales de enfermería de los Equipos de Atención Primaria de la Comunidad Valenciana. Entre los resultados obtenidos en este trabajo podemos destacar que la estandarización de los objetivos y criterios de evaluación presenta un efecto negativo sobre el conflicto y sobre la ambigüedad de rol, mientras que tiene un efecto positivo sobre la satisfacción laboral. Además de estos efectos directos, la estandarización tiene efectos indirectos a través de la ambigüedad de rol, siendo negativos sobre la tensión y positivos sobre la satisfacción laboral.

Estas relaciones resaltan la importancia de la estandarización de los objetivos y criterios de evaluación respecto a las disfunciones del episodio de rol estudiadas, ejerciendo influencia tanto directa como indirecta sobre la satisfacción laboral.

Es interesante destacar que en este modelo sólo la ambigüedad de rol presenta efectos estadísticamente significativos sobre la tensión laboral y la satisfacción laboral, no ocurriendo lo mismo con el conflicto de rol. Por último, consideramos importante el resultado obtenido en la relación establecida entre la tensión laboral y la propensión al abandono, ejerciendo un efecto directo, positivo y significativo, mientras que el efecto de la satisfacción laboral sobre la propensión al abandono no resulta significativo. Estos resultado son contradictorios con los obtenidos en la mayor parte de estudios realizados sobre el tema, únicamente Jackson (1983-83), considerando ambas relaciones causales simultáneamente, obtuvo resultados estadísticamente significativos.

Como indican Lloret et al. (1992) en los estudios precedentes los modelos considerados, al no incluir la relación entre la tensión y la propensión al abandono, podrían presentar el problema de falta de autocontenido y producir una 
sobreestimación del efecto de la satisfacción laboral sobre la propensión al abandono. Por otra parte, los autores señalan la necesidad de nuevas investigaciones que analicen las relaciones por ellos encontradas en diferentes muestras con el fin de determinar si el modelo es una buena representación del conjunto de relaciones estudiadas.

Siguiendo esta línea de investigación en nuestro trabajo nos proponemos como objetivo contrastar empíricamente el modelo de antecedentes y consecuentes del estrés de rol formulado por Lloret (1991) y representado en la figura 1.

Figura 1.- Modelo causal propuesto

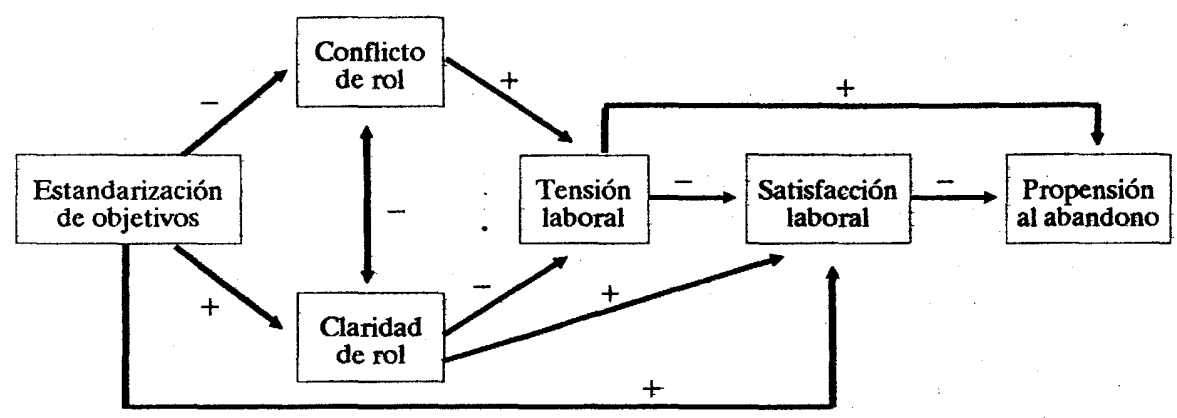

El modelo propuesto en nuestro estudio reproduce, en términos generales, las relaciones consideradas en el modelo formulado por Lloret (1991), salvo algunas excepciones que son las siguientes:

1. Como variable antecedente del conflicto y la ambigüedad de rol hemos incluido unicamente la estandarización de objetivos, que tiene un efecto directo y positivo sobre la claridad de rol y la satisfacción laboral, y un efecto directo y negativo sobre el conflicto de rol.

2. El conflicto de rol tiene un efecto directo y positivo sobre la tensión laboral, esta relación no se mantiene en el modelo de Lloret (1991), sin embargo, en estudios previos este resultado obtiene un apoyo con- 
sistente (Bedeian y Armenakis, 1981; Kemery et al., 1985; Peiró et al., 1985).

3. La satisfacción laboral tiene un efecto directo y negativo sobre la propensión al abandono. En el modelo de Lloret (1991), el efecto de la satisfacción laboral sobre la propensión al abandono no se mantiene al considerar el efecto de la tensión laboral sobre dicha variable. Sin embargo, todos los estudios precedentes dan apoyo empírico a esta relación. Unicamente el trabajo de Jakcson (1983-84) considera ambas relaciones, las satisfacción laboral y la tensión laboral sobre la propensión al abandono, resultando significativas.

\section{METODO}

\section{Muestra.}

La muestra está compuesta por 178 profesionales sanitarios que desempeñan su rol en hospitales, clínicas y Centros de Atención Primaria de la Comunidad Valenciana, tanto en la Seguridad Social como en organizaciones privadas.

En este estudio hemos incluido únicamente médicos y personal de enfermería, quedando excluidos otros profesionales no directamente relacionados con el cuidado de la salud, tales como celadores y administrativos.

La descripción de la muestra es la siguiente:

En función del rol profesional 90 son enfermeras (50.6\%) y 88 son médicos (49.4\%).

Respecto al nivel de intervención en el que actúan estos profesionales, 92 trabajan en centros de atención primaria (51.7\%) y 86 en hospitales (48.3\%).

En relación al sexo, la muestra está equiparada ya que 89 son mujeres (50\%) y 89 son hombres (50\%).

Atendiendo a la edad, 16 son menores de 25 años (9\%); 53 tienen edades comprendidas entre 26 y 30 años (29.8\%); 60 entre 31 y 35 años (33.7\%); 28 entre 36 y 40 años (15.7\%); 16 entre 41 y 50 años (9\%) y 5 entre 51 y 60 años (2.8\%).

Respecto a la experiencia profesional, 69 sujetos llevaban trabajando menos de 1 año en la empresa (38.8\%); 47 llevaban trabajando entre 1 y 3 años (26.4\%); 30 entre 3 y 6 años (20.2\%); 19 entre 6 y 9 años (10.7\%) y 7 más de 9 años (3.9\%). 


\section{Instrumentos de Medida.}

Se han utilizado como instrumentos de medida una serie de cuestionarios de autoinforme, elaborados o adaptados por la Unida de Investigación de Psicología de la Organización y del Trabajo (U.I.P.O.T.) de la Universidad de Valencia para la realización de un estudio de los profesionales que desempeñan su rol en los Equipos de Atención Primaria de la Comunitat Valenciana. En la Tabla 1 se encuentran los resultados descriptivos de los distintos cuestionarios.

Tabla 1.- Resultados descriptivos y correlacionales entre las variables

\begin{tabular}{|c|c|c|c|c|c|c|c|c|c|}
\hline & $\overline{\mathbf{x}}$ & DT & $\mathbf{R}$ & 1 & 2 & 3 & 4 & 5 & 6 \\
\hline $\begin{array}{l}\text { 1. Satisfacción } \\
\text { laboral }\end{array}$ & 4.05 & 1.10 & $1-7$ & 1 & & & & & \\
\hline 2. Conflicto de rol & 3.69 & 1.37 & $1-7$ & -.39 & 1 & & & & \\
\hline 3. Claridad de rol & 5.02 & 1.40 & $1-7$ & .68 & -.49 & 1 & & & \\
\hline $\begin{array}{l}\text { 4. Estandarización } \\
\text { de objetivos }\end{array}$ & 2.83 & 0.89 & $1-5$ & .54 & -.29 & .65 & 1 & & \\
\hline 5. Tensión laboral & 1.92 & 0.66 & $1-5$ & -.46 & .24 & -.35 & -.31 & 1 & \\
\hline $\begin{array}{l}\text { 6. Propensión al } \\
\text { abandono }\end{array}$ & 2.26 & 1.00 & $1-5$ & -.25 & .17 & -.18 & -.09 & .37 & 1 \\
\hline
\end{tabular}

Para medir la Tensión Laboral hemos utilizado el cuestionario de Tensión Laboral validado por Meliá et al. (1987). Es un instrumento de seis items con alternativas de respuesta tipo Likert de cinco puntos de anclaje que van desde 1 "nunca" a 5 "casi siempre o siempre". La puntuación total en la escala se obtiene con el promedio de la suma de las puntuaciones en cada uno de los items. El contenido de los ítems hace referencia a trastornos psicosomáticos o sentimientos negativos que puede experimentar el sujeto en su puesto de trabajo, tales como nerviosismo, ansiedad o insomnio. El cuestionario nos indica el grado en el que sujeto experimenta estas situaciones.

El instrumento que mide la Propensión al Abandono del puesto de trabajo y de la organización está compuesto solamente por dos ítems que proceden de las investigaciones de Kahn y col. (1964). Las alternativas de respuesta son cinco, desde 1. "muy en desacuerdo" hasta 5. "muy de acuerdo". El primer ítem es inverso y su puntuación transformada nos indica el grado de propensión a abandonar el puesto de trabajo, el ítem es: "en igualdad de condiciones (económicas, estatus, horario...) prefiero trabajar en mi unidad de trabajo a hacerlo en otras unidades del mismo centro o nivel asistencial". El contenido del segundo ítem hace referencia a la propensión a abandonar la organización o centro de 
trabajo, es el siguiente: "en igualdad de condiciones (económicas, estatus, horario....) prefiero trabajar en una unidad de otro nivel asistencial al que trabajo actualmente".

El cuestionario utilizado para medir la estandarización de la tarea consta de tres ítems que proceden del Instrumento de Evaluación Organizacional (OAI) elaborado por Van de Ven y Ferry (1980). Es una escala con alternativas de respuesta tipo Likert con cinco puntos de anclaje. El contenido de los ítems hace referencia a la claridad con que el sujeto conoce el nivel de rendimiento que se espera de él; la claridad con que se especifica la descripción del puesto de trabajo, las normas o niveles de rendimiento que han de ser evaluados y la dificultad para lograr los niveles y cumplir las normas de rendimiento.

La escala de Conflicto de Rol consta de seis ítems, cinco de los cuales proceden del instrumento elaborado por Rizzo, House y Lirtzman (1970) y el ítem restante, "recibo demandas de los usuarios incompatibles con la normativa existente", ha sido creado específicamente para los estudios realizados con muestras de profesionales sanitarios. El formato de respuesta es una escala tipo Likert con 7 alternativas de respuesta, desde 1. "completamente en desacuerdo" a 7. "completamente de acuerdo". La puntuación total del sujeto se obtiene promediando la suma de las puntuaciones en cada ítem.

El contenido de los ítems hace referencia a diferentes tipos de conflictos que puede experimentar el sujeto en su puesto de trabajo: conflicto personarol, conflicto medios-fines y conflicto inter-emisores, aunque en el presente estudio únicamente se obtienen un índice global de este constructo.

El instrumento que mide la Ambigüedad de Rol consta de siete ítems, seis proceden de la escala creada por Rizzo, House y Lirtzman (1970) y el séptimo "los procedimientos que he de emplear para desempeñar mi trabajo son claros" ha sido especialmente creado para los estudios con profesionales sanitarios. Al igual que el anterior posee siete alternativas de respuesta, desde 1. "completamente en desacuerdo" a 7. "completamente de acuerdo". Mediante una transformación realizada en las puntuaciones, en nuestro estudio la puntuación obtenida por el sujeto nos indica la claridad de rol experimentada por el mismo, en lugar de la ambigüedad de rol.

Finalmente, la Satisfacción Laboral ha sido medida con el Cuestionario Modular de Satisfacción Laboral de Profesionales Sanitarios (B.O.P.) (Bravo et al. 1991). Este instrumento que evalúa múltiples facetas de la satisfacción laboral consta de tres módulos (Básico, Organización y Público) aplicables en función de las características laborales específicas de los profesionales de la salud. En este estudio, hemos aplicado el módulo Básico, destinado a todo tipo de profesionales sanitarios, independientemente del contexto laboral en el que 
se encuentren. Consta de 32 items, con siete alternativas de respuesta que van desde 1. "muy insatisfecho" a 7. "muy satisfecho".

La factorización de los 32 ítems de éste módulo ofrece una estructura de seis factores relacionados con la satisfacción intrínseca y posibilidades de formación y desarrollo, con las retribuciones e incentivos, con el ambiente físico y la disponibilidad de personal auxiliar, con la claridad y autonomía de tareas, con los aspectos temporales y la sobrecarga de trabajo y en las relaciones humanas con pacientes y compañeros. En el presente estudio hemos utilizado un índice global del constructo.

\section{Análisis de Datos.}

Con el objeto de examinar el modelo hipotetizado (figura 2) hemos utilizado el paquete estadístico LISREL VII, calculando los parámetros estructurales por estimación de máxima verosimilitud. Con este tipo de análisis realizamos tanto un contraste empírico analítico de cada una de las relaciones causales hipotetizadas por separado, como un contraste empírico global que evalúa la bondad de ajuste a los datos de la secuencia causal hipotetizada.

\section{Figura 2.- Modelo causal propuesto}

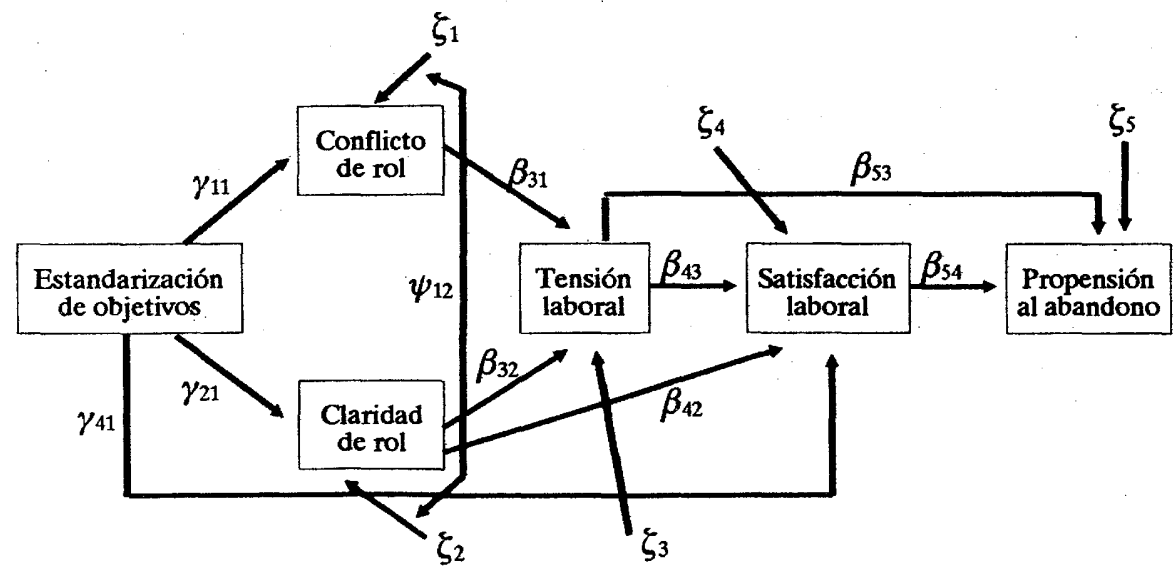

Para el contraste empírico analítico utilizamos los coeficientes estructurales obtenidos en función de la significación estadística de los mismos. 
Para el contraste empírico global LISREL VII proporciona una serie de índices que evalúan el ajuste global del modelo. Estos son: 1) el índice de bondad de ajuste ji-cuadrado $\left(\mathrm{X}^{2}\right)$ y su probabilidad asociada, que indica el grado de ajuste con que el modelo propuesto reproduce los datos observados. 2) El índice de bondad de ajuste (GFI), indica la cantidad global de variación que explica el modelo propuesto. 3) El índice de bondad de ajuste ajustado (AGFI), ajusta el índice anterior a los grados de libertad del modelo propuesto, el GFI y el AGFI varían de cero a uno, siendo 1 el ajuste perfecto. 4) La raíz de la medias cuadráticas residuales (RSMR) que es una medida del promedio de los residuales ajustados. 5) El cociente $\mathrm{X}^{2} / \mathrm{gl}$. 6) Y los índices "delta" ( $\Delta$ incremental fit index) y "rho" ( $\rho$ non normative fit index) que ofrecen información acerca del grado en que el modelo puede ser mejorado.

El índice de bondad de ajuste ji-cuadrado, indica la diferencia entre la matriz de covarianza observada y la que el modelo especificado predice, pero presenta el inconveniente de que es sensible al tamaño de la muestra (Jöreskog, 1978; Jöreskog y Sörbom, 1989), por lo que al aumentar el tamaño de la muestra, también aumenta la probabilidad de rechazar el modelo. Debido a ello se recomienda calcular otros índices de bondad de ajuste (Bentler y Bonnet, 1980).

Tales índices son el cociente X2/gl (Jöreskog, 1969)., cuando este cociente es menor que 10 se considera un buen ajuste (Boruch y Wolings,1970). Y según Carmines y Mclver (1981) si es menor que 2 se considera indicador de un ajuste excelente. En este sentido, también utilizamos los índices "rho" (Tucket y Lewis, 1973) y "delta" (Bentler y Bonnet, 1980), siendo el primero el menos afectado por el tamaño de la muestra. Valores por encima de .90 nos indican que el modelo propuesto es aceptable, y por debajo que el modelo puede ser mejorado. Con muestras superiores a 100 sujetos, Bearden, Sharma y Teel (1982) afirman que un índice "delta" menor de .95 indica un pobre ajuste.

Es recomendable realizar el análisis de todos estos índices, pues la evaluación de la bondad de ajuste debe basarse en un criterio múltiple (Bentler y Bonett, 1980).

\section{RESULTADOS}

Los resultados de lo índices que evalúan la bondad de ajuste del modelo se presentan en la tabla 2. En ella puede observarse que el índice ji-cuadrado $\left(X^{2}: 5.64\right)$ calculado con cinco grados de libertad no es estadísticamente significativo (p: 0.342 ), esto indica que el modelo hipotetizado es una posible repre- 
sentación de las relaciones de influencias entre las variables en una población. El cociente $\mathrm{X}^{2} / \mathrm{gl}$ alcanza un valor de 1.12 , lo que indica un buen ajuste según el criterio de Carmines y Mclver (1981). Los índices GFI, AGFI y RMSR en este caso también son indicadores de un buen ajuste entre el modelo propuesto y los datos. El Índice "delta" alcanza un valor de .98 y el índice "rho" de .95 .

Tabla 2.- Indices de bondad de ajuste para el modelo hipotetizado

\begin{tabular}{ccccccccc}
$\mathbf{X}^{2}$ & $\mathbf{D F}$ & $\mathbf{P}$ & $\mathbf{G F I}$ & AGFI & RMRS & $\Delta$ & $\rho$ & $\mathbf{H}^{2} / \mathbf{I}$ \\
\hline \hline 5.64 & 5 & 0.342 & .99 & .95 & .027 & .98 & .95 & 1.12 \\
\hline
\end{tabular}

A nivel de evaluación analítica del modelo, hemos analizado los coeficientes estructurales, estos resultados se encuentran en la figura 3, donde se muestran los valores de máxima probabilidad para los coeficientes estructurales estimados. En primer lugar, en todos los casos los coeficientes presentan el signo hipotetizado. Por otra parte, dos de ellos no alcanzan valores de significación estadística, es el caso del efecto del conflicto de rol sobre la tensión laboral $\left(\beta_{31}: 0.09\right)$ y el efecto de la satisfacción laboral sobre la propensión al abandono $\left(\beta_{54:}\right.$ 0.092). El efecto de la estandarización de los objetivos sobre la satisfacción laboral alcanza valores muy próximos al nivel de significación estadística ( $\gamma_{41}: 0.134$, p: 0.06 ), por lo que hemos mantenido esta relación.

\section{Figura 3.- Resultados del modelo causal propuesto}

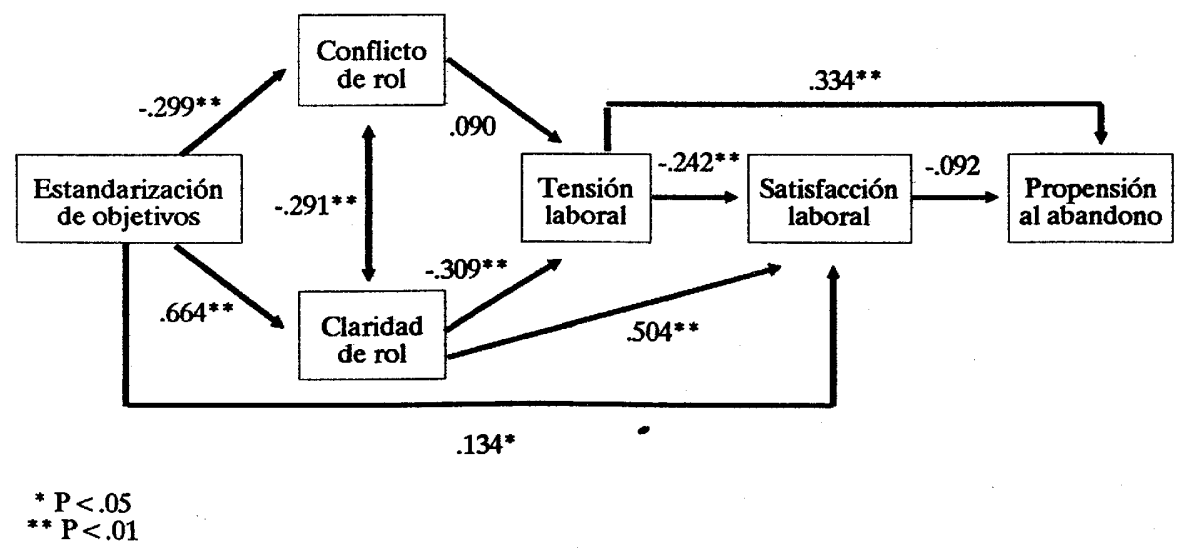


Respecto a los índices de modificación del modelo ofrecidos por el LISREL VII, en ningún caso nos indica la necesidad de incluir nuevas relaciones en el modelo propuesto.

Ya que dos de los coeficientes del modelo hipotetizado no alcanzan valores de significación estadística, proponemos un modelo revisado en el cual no se incluyen dichos coeficientes.

Figura 4.- Modelo causal revisado

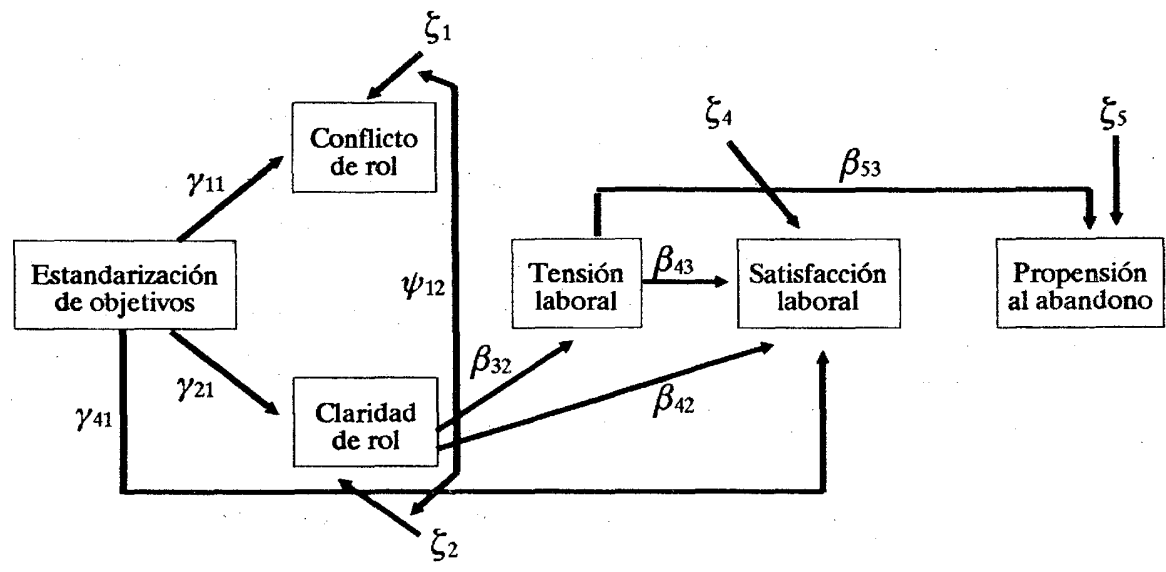

Tabla 3.- Indices de bondad de ajuste para el modelo revisado

\begin{tabular}{ccccccccc}
$\mathbf{X}^{2}$ & DF & $\mathbf{P}$ & GFI & AGFI & RMRS & $\Delta$ & $\rho$ & $\mathbf{H}^{2} / \mathbf{l}$ \\
\hline 8.29 & 7 & 0.308 & .98 & .95 & .049 & .97 & .95 & 1.17 \\
\hline
\end{tabular}

El modelo revisado, en el que no aparecen los efectos que no alcanzaban el nivel de significación estadística, se presenta en la figura 4. Los valores obtenidos para los índices de bondad de ajuste se recogen en la tabla 3. El índice jicuadrado $\left(\mathrm{X}^{2}: 8.29\right)$ calculado en este caso con siete grados de libertad, no es estadísticamente significativo (p: 0.308 ), por lo que el modelo hipotetizado es una posible representación de las relaciones de influencias entre las variables en una población. El cociente $\mathrm{X}^{2} / \mathrm{gl}$ presenta un valor de 1.17 , situándose nue- 
vamente bajo el criterio mantenido por Carmines y Mclver (1981), podemos considerar que nuestros resultados muestran un ajuste excelente. Los índices GFI, AGFI y RMSR indican un ajuste satisfactorio y los índices "delta" y "rho" son de .97 y .95 respectivamente. En general, los cambios introducidos en el modelo mantienen todos los índices de bondad en un nivel similar al modelo inicialmente propuesto, alcanzando resultados que indican un buen ajuste general del modelo.

Figura 5.- Resultados del modelo causal revisado

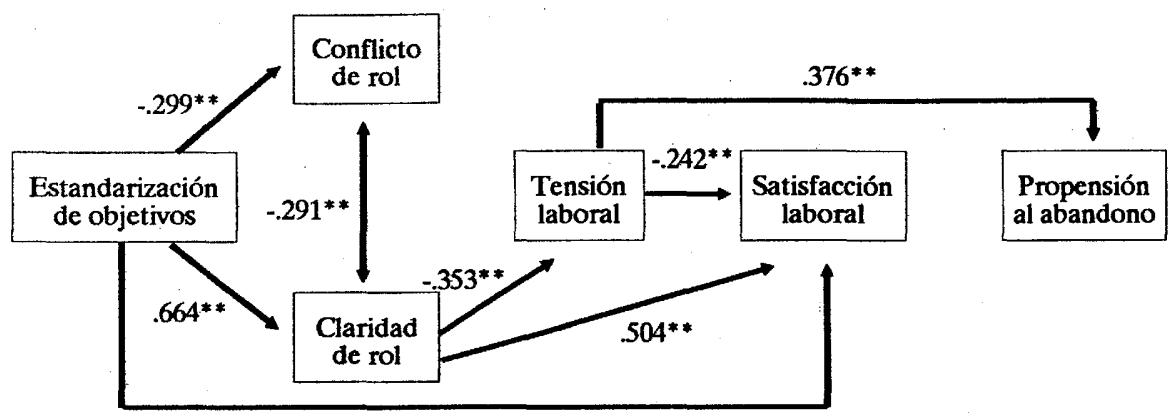

$.134^{*}$

$* \mathrm{P}<.05$

$* * P<.01$

En la figura 5 se ofrecen los valores de máxima probabilidad para los coeficientes estructurales estimados en el modelo revisado. Todos los coeficientes estructurales incluidos en el modelo son estadísticamente significativos. Los índices de modificación no alcanzan valores que nos indiquen la inclusión de nuevas relaciones causales.

\section{DISCUSION Y CONCLUSIONES}

Como ya hemos indicado el objetivo de este estudio es la contrastación empírica del modelo de antecedentes y consecuentes del estrés de rol propuesto por Lloret (1991) para profesionales sanitarios. El modelo propuesto inicial- 
mente presentaba un buen ajuste con los datos. El contraste empírico analítico muestra que todos los coeficientes estructurales incluidos en el modelo presentaban valores estadísticamente significativos excepto los correspondientes al efecto del conflicto de rol sobre la tensión laboral y el correspondiente a la satisfacción laboral sobre la propensión al abandono. En base a estos resultados hemos planteado de nuevo el modelo excluyendo dichos efectos no significativos. El resultado ha sido un modelo más parsimonioso que presenta un buen ajuste con los datos observados. Este modelo reproduce las relaciones encontradas por Lloret (1991) para profesionales sanitarios que han sido consideradas en nuestro modelo, dando apoyo empírico a dicho modelo.

Pasamos a comentar a continuación las relaciones causales implicadas en el modelo. La estandarización de los objetivos presenta un efecto directo y negativo sobre el conflicto de rol, de manera que incrementos en la estandarización de los objetivos producirán decrementos en el conflicto de rol percibido. Por otra parte, la estandarización de los objetivos ejerce un efecto directo y positivo sobre la claridad de rol, de manera que incrementos en la misma producirán a su vez incrementos en la claridad de rol percibida. Por último, la estandarización también presenta un efecto directo positivo sobre la satisfacción laboral, de manera que incrementos en la primera producirán incrementos en la segunda. Estos resultados son coherentes con los encontrados por Lloret (1991).

El conflicto de rol no presenta un efecto significativo sobre la tensión laboral. Frente a otros estudios en los que esta relación ha tenido confirmación empírica (Bedeian y Armenakis, 1981; Peiró y col., 1985; Kemery y col., 1985) nuestros resultados coinciden con los de Lloret (1991) no obteniéndose confirmación empírica de dicha relación.

Sin embargo, la claridad de rol se tiene un efecto directo negativo sobre la tensión laboral, de tal forma que incrementos en la claridad de rol percibida producirán decrementos en la tensión laboral experimentada, esta relación aparece en numerosos trabajos realizados con profesionales de la salud (Bedeian y Armenakis, 1981; Kemery y col., 1985; González y col., 1989; Lloret. 1991). También la claridad de rol ejerce un efecto directo positivo sobre la satisfacción laboral, de manera que niveles elevados de claridad de rol percibida conllevan también niveles elevados de satisfacción laboral experimentada, estos resultados son coherentes con los obtenidos por Bedeian y Armenakis (1981) y Lloret (1991) en profesionales sanitarios. El conflicto de rol y la claridad de rol presentan una correlación negativa, resultado que no aparecía en el modelo de Lloret (1991).

La tensión laboral ejerce un efecto directo y negativo sobre la satisfacción laboral, este resultado aparece también en los estudios realizados por Bedeian 
y Armenakis (1981), Kemery y col., (1985) y Lloret (1991). Un resultado interesante es el efecto directo positivo de la tensión laboral sobre la propensión al abandono que aparece también en dos estudios, el de Jackson (1983) y el de Lloret (1991), ya que en el resto de los estudios este nexo no había sido introducido pues la inclusión del mismo en los modelos de Bedeian y Armenakis (1981) y Kemery y col., (1985) convertirán los modelos hipotetizados en modelos completos e imposibles de falsear. La inclusión de esta relación en el modelo anula un efecto que ha aparecido en numerosos estudios, es el efecto de la satisfacción laboral sobre la propensión al abandono. Como señala Lloret $(1991,156)$ "Estos resultado indican que estábamos cometiendo un error de especificación al no introducir este nexo. La tensión laboral aumenta la propensión a abandonar el trabajo y, de forma simultánea, disminuye la satisfacción laboral. Ambos efectos son independientes y no secuenciales".

Nuestro estudio muestra, que el conflicto y la ambigüedad de rol tienen patrones diferenciados de efectos disfuncionales, por lo tanto se señala la necesidad de avanzar en el sentido de formular hipótesis diferenciadas para cada una de estas variables (Jackson y Schuler, 1985; Lloret, 1991).

Hay que señalar que el modelo planteado no incluye todos los posibles antecedentes de las variables endógenas, lo que se denomina como falta de autocontaminación (James et al, 1982), y ello puede llevar a coeficientes estructurales sesgados. Por otra parte, obtener una serie de índices que nos indican que el modelo presenta un buen ajuste, no quiere decir que sea el único ni el mejor modelo posible, sino que las mismas variables situadas en distinto orden causal pueden presentar también un buen ajuste (Fry et al., 1986), este tipo de análisis son más útiles para rechazar un modelo que para afirma un modelo como válido (Peiró et al., 1990).

\section{REFERENCIAS}

Bearden WO, Sharma S y Teel JE. Sample size effects on chi-square and other statistics used in evaluating causal models. J Marketing Res 1982, 19, 425-430.

Bedeian AG y Armenakis A. A path-analytic study of the consequences of role conflict and ambiguity. Academy of Management Journal 1981, 24, 2, 417-424.

Bentler PM y Bonnet DG. Significance test and goodness of fit in the analysis of covariance structure. Psychol Bull 1980, 80, 588-606. 
Bravo MJ, Peiró JM y Zurriaga R. El cuestionario modular de satisfacción laboral de profesionales de la salud (B.O.P.). Psiquis 1991, 12, 51-62.

Carmines E y McIver J. Analyzing models with unobserved variables: Analysis of covariance structures. En: G Bohrnstedt y E Borgatta, (editores). Social Measurement: Current Issues. Beverly Hills: Sage, 1981.

Cummings RC. Job stress and the buffering effect of supervisory support. Group and Organization Studies 1990, 15(1), 92-104.

Fisher CD y Gitelson R. A meta-analysis of the correlates of role conflict and ambiguity. J Appl Psychol 1983, 68(2), 320-333.

Fry LW, Futrell CM, Parasuraman A y Chmielewski MA. An analysis of alternative causal models of salesperson role perceptions and work-related attitudes.J Marketing Res 1986, 23, 153-163.

Gonzalez-Romá V. Un modelo causal de los antecedentes y las consecuencias del estrés de rol [tesis doctoral]. Universidad de Valencia, 1989.

Gonzalez-Romá V, Meliá JL, Peiró JM, Zurriaga R y Luque O. A causal model of the effects of perceived role stress: a multisample test. Paper presentado al 4th West European Congress on the Psychology of Work and Organisation. Cambridge, U.K., 1989.

Jackson SE. Participation in decision making as a strategy for reducing job-related strain. J Appl Psychol 1983, 68(1), 3-19.

Jackson SE. Correction to "Participation in decision making as a strategy for reducing job-related strain". J Appl Psychol 1984, 69(3), 546-547.

Jackson SE y Schuler RS. A meta-analysis and conceptual critique of research on role ambiguity and role conflict in work sttings. Organizational Behavior and Human Decision Processes 1985, 36, 16-78.

James LR, Mulaik SA y Brett JM. Causal analysis. Assumptions, models y data. Beverly Hills: Sage, 1982.

Jöreskog KG. Structural analysis of covariance y correlation matrices. Psychometrika 1978, 43, 443-447. 
Jöreskog KG y Sörbom D. LISREL VII. User's referende guide. Mooresville: Scientific Software, Inc., 1989.

Kahn RL, Wolfe DM, Quinn RP, Snoek JD y Rosenthal RA. Organizational Stress: studies in role conflict and ambiguity. New York: Wiley, 1964.

Kemery ER, Bedeian AG, Mossholder KW y Touliatos J. Outcomes of role stress: a multisample constructive replication. Academy of Management Joumal 1985, 28(2), 363-375.

Lloret S. El estrés de rol en enfermeras: un modelo causal [tesis de licenciatura]. Universidad de Valencia, 1991.

Lloret S, Gonzalez-Romá V y Peiró JM. El estrés de rol en enfermeras: un modelo causal. Revista de Psicología General y Aplicada [en prensa, 1992].

Meliá JL, Peiró JM, Zornoza A, Sanz, Morte y Zurriaga R. Una escala para la evaluación de la Tensión de Rol: estructura factorial, fiabilidad y validez. II Congreso de Evaluación Psicológica. Septiembre, Madrid, 1987.

Oliver RL y Brief AP. Determinants and consequences of role conflict and role ambiguity among retail sales managers. Joumal of Retailing 1977-78, 53(4), 4758.

Peiró JM, Gonzalez-Romá V, Valcarcel P y Ramos J. Professionals' disenchantment a new implemented model of primary health care: a structural equations model. Trabajo presentado al II Workshop on the Psychology of Work in Hospitals, 5-7 Abril, Valencia, 1990.

Peiró JM, Meliá JL y Gonzalez-Romá V. La incidencia del conflicto y la ambigfedad de rol sobre la propensión al abandono y la satisfacción laboral. Actas del II Congreso Nacional de Psicología del Trabajo, 305-333, Terrassa, 1985.

Rizzo JR, House RJ y Lirtzman SI. Rol conflict and ambiguity in complex organizations. Administrative Science Quarterly 1970, 15, 150-163.

Tucker LR y Lewis C. The reliability index for maximun likelihood factor analysis. Psychometrika 1973, 38, 1-10. 
Van de Ven AH y Ferry DL. Measuring and Assessing Organizations. New York: Wiley and Sons, 1980. 